\title{
Riesgo psicosocial y desempeño académico: un análisis en los colegios de la Policía Nacional adscritos a la ciudad de Bogotá, D. C.
}

\author{
Psychosocial risk and academic performance:An analysis in the National Police \\ schools affiliated with the city of Bogotá, D.C.
}

\section{Risco psicossocial e desempenho acadêmico: uma análise nas escolas da Polícia Nacional na cidade de Bogotá, D.C.}

\section{Adriana Carolina Segura Baracaldo ${ }^{\mathrm{a}^{*}}$ | Leidy Johanna Rojas Landínez $\mathrm{z}^{\mathrm{b}}$ Christian Camilo Benavides Acosta $^{c}$}

${ }^{a}$ https://orcid.org/0000-0002-II80-283X

${ }^{b}$ https://orcid.org/0000-0002-3131-7516

c https://orcid.org/0000-0002-I I 96-9648

Policía Nacional de Colombia, Colombia

- Fecha de recepción: 2020-0I-I5

- Fecha concepto de evaluación: 2020-04-25

- Fecha de aprobación: 2020-05-09 https://doi.org//0.22335/rlct.v12i2.1/29
Para citar este artículo / To reference this article / Para citar este artigo: Segura Baracaldo, A. C., Rojas Landínez, L. J., \& Benavides Acosta, C. C. (2020). Riesgo psicosocial y desempeño académico: un análisis en los colegios de la Policía Nacional adscritos a la ciudad de Bogotá, D. C. Revista Logos Ciencia \& Tecnología, I 2(2), 44-56. https://doi.org// 0.22335/rlct. v12i2. 1129

\section{RESUMEN}

Según el Análisis de Riesgos Psicosociales del 2018, realizado por el Observatorio de la Familia Policial de la Dirección de Bienestar Social, se presentaron 2.229 casos de riesgo psicosocial en los 22 colegios, con un incremento del $50,5 \%$ respecto al año anterior, siendo recurrentes en los informes anuales en la Institución. Por lo anterior, este estudio pretende determinar la relación que existe entre el riesgo psicosocial y el desempeño académico en adolescentes con edades entre 13 y 15 años en Bogotá, usando, como herramienta de recolección de información, el Cuestionario para la Evaluación de Problemas en Adolescentes Q-PAD, para identificar los índices de insatisfacción corporal, ansiedad, depresión, abuso de sustancias, problemas interpersonales, problemas familiares, incertidumbre con el futuro, riesgo psicosocial y autoestima; $y$ el desempeño académico reportado por los colegios. Esta investigación de tipo cuantitativo no experimental-correlacional, contó con la participación de 650 adolescentes de los grados $8^{\circ}, 9^{\circ}$ y $10^{\circ}$ en tres colegios de la ciudad de Bogotá adscritos a la Policía Nacional. Los resultados mostraron que el $9 \%$ de los adolescentes presentó un alto índice de riesgo psicosocial. Respecto al desempeño académico, aunque se evidenció una correlación con el riesgo psicosocial, este no fue significativo para determinar causalidad; sin embargo, sí se encontró que las mujeres presentan mayor riesgo psicosocial en comparación con los hombres. Por medio del análisis estadístico Kruskal-Wallis, se halló una asociación con el desempeño académico y los tipos de familia, pues los integrantes de familias nucleares muestran un mejor rendimiento académico que los pertenecientes al tipo de familia extensa. Finalmente, con el estadístico descriptivo se identificó que la tipología familiar presenta correlación significativa con el riesgo psicosocial, y muestra que los estudiantes que provienen de familias extensas presentan menor riesgo, que los que pertenecen a familias recompuestas.

Palabras clave: psicología de la educación, rendimiento escolar, adolescencia, autoestima, policía 


\section{ABSTRACT}

According to the 2018 Psychosocial Risk Analysis, carried out by the Police Family Observatory of the Social Welfare Directorate, 2,229 cases of psychosocial risk were detected in the 22 schools, an increase of $50.5 \%$ from the previous year, a figure which has been recurring in the institution's annual reports. Therefore, this study aims to determine the relationship between psychosocial risk and academic performance in adolescents between the ages of 13 and 15 in Bogotá, using the Questionnaire, Q-PAD, for the Evaluation of Problems in Adolescents as a tool for collecting information in order to identify indices of body dissatisfaction, anxiety, depression, substance abuse, interpersonal problems, family problems, uncertainty about the future, psychosocial risk and self-esteem, as well as the academic performance reported by the schools. 650 adolescents from the 8th, 9th and 10th grades of three schools assigned to the National Police in Bogota participated in this non-experimental-correlational quantitative research. The results showed that $9 \%$ of the adolescents presented a high index of psychosocial risk. Regarding academic performance, although a correlation with psychosocial risk was evidenced, it was not significant to determine causality; however, it was found that women have a higher psychosocial risk compared to men. Through the Kruskal-Wallis statistical analysis, an association was found between academic performance and family types, since members of nuclear families show better academic performance than those belonging to extended families. Finally, with the descriptive statistic, it was identified that the family typology presents a significant correlation with the psychosocial risk, indicating that students which come from extended families have a lower risk than those who belong to recomposed families.

Keywords: educational psychology, school performance, adolescence, self-esteem, police

\section{RESUMO}

De acordo com a Análise de Risco Psicossocial de 2018, realizada pelo Observatório da Família Policial da Diretoria de Assistência Social, 2.229 casos de risco psicossocial foram apresentados nas 22 escolas, com aumento de $50,5 \%$ em relação ao ano anterior, sendo recorrente nos relatórios anuais da Instituição. Portanto, este estudo tem como objetivo determinar a relação entre risco psicossocial e desempenho acadêmico em adolescentes entre 13 e 15 anos em Bogotá, utilizando o Questionário de Avaliação de Problemas em Adolescentes como ferramenta para coleta de informações. Q-PAD, para identificar índices de insatisfação corporal, ansiedade, depressão, abuso de substâncias, problemas interpessoais, problemas familiares, incerteza com o futuro, risco psicossocial e autoestima; e o desempenho acadêmico relatado pelas escolas. Esta pesquisa quantitativa, não experimental-correlacional, contou com a participação de 650 adolescentes da $8^{\mathrm{a}}, 9^{\mathrm{a}}$ e $10^{\mathrm{a}}$ ano em três escolas da cidade de Bogotá, pertencentes à Polícia Nacional. Os resultados mostraram que $9 \%$ dos adolescentes apresentaram um alto índice de risco psicossocial. Em relação ao desempenho acadêmico, embora tenha sido evidenciada correlação com risco psicossocial, não foi significativo para determinar causalidade; no entanto, verificou-se que as mulheres apresentam maior risco psicossocial comparadas aos homens. Através da análise estatística de Kruskal-Wallis, foi encontrada uma associação com o desempenho acadêmico e os tipos de família, uma vez que membros de famílias nucleares apresentam melhor desempenho acadêmico do que aqueles pertencentes ao tipo de família extensas. Por fim, com a estatística descritiva, identificou-se que a tipologia familiar apresenta correlação significativa com o risco psicossocial, e mostra que os alunos provenientes de famílias extensas apresentam menor risco do que aqueles pertencentes a famílias recompostas.

Palavras-chave: psicologia educacional, rendimento escolar, adolescência, auto estima, polícia

El estudio "Riesgo psicosocial y desempeño académico: un análisis en los colegios de la Policía Nacional de la ciudad de Bogotá, D. C., brinda herramientas de detección y orientación efectiva del riesgo psicosocial para el mejoramiento de la calidad educativa en adolescentes, permitiendo prevenir de esta forma, la materialización de problemas, tales como el consumo de sustancias, la depresión, la ansiedad, la insatisfacción corporal, la incertidumbre hacia el futuro, 
el embarazo en adolescentes, el bullying, el suicidio, entre otras afectaciones.

Se realizó un análisis cuantitativo correlacional que permite una aproximación hacia la comprensión del riesgo psicosocial con relación al desempeño académico, desde un contexto psicoeducativo en adolescentes entre 13 y 15 años de edad. Para esto se parte del registro de información de análisis de riesgo psicosocial, realizado por el Observatorio de la Familia Policial de la Dirección de Bienestar Social de la Policía Nacional de Colombia, de acuerdo con la fuente de información del grupo de educación que lidera los 22 colegios de la Institución.

Por lo anterior, se da la necesidad de investigar el riesgo psicosocial en los adolescentes, puesto que esta etapa desde la psicología del desarrollo, es donde el ser humano busca reafirmar su identidad psicosocial desde la relación con sus pares y el entorno, conllevando muchas veces a que los adolescentes se encuentren en situaciones que los llevan a conductas de exposición y de riesgo, que pueden comprometer su salud física y mental, con el objetivo de ser aceptados socialmente e incorporarse a un grupo específico. Asimismo, esta detección de riesgo psicosocial, en relación con el desempeño académico, resulta esencial para el diseño de estrategias curriculares y de tipo preventivo con la corresponsabilidad de la familia, los docentes y directivos, de forma integral que redunde en el bienestar y calidad de vida de los jóvenes y las familias policiales.

\section{Problema de investigación}

La Dirección de Bienestar Social, por medio del Observatorio de la Familia, consolida información de los riesgos psicosociales provenientes de diferentes fuentes, con el propósito de generar planes, programas y proyectos que fortalezcan y contribuyan a la sostenibilidad de las familias policiales. Estas fuentes de información incluye a los 22 colegios de la Policía Nacional, los cuales aportan información sobre diversos problemas que afectan a los estudiantes $y$, por ende, tienen una incidencia directa en las familias policiales.

En el consolidado de Análisis de Riesgos Psicosociales del 2018, emitido por el Observatorio de la Familia Policial, se evidencia en los colegios de la Policía Nacional un incremento considerable de las problemáticas psicosociales en los estudiantes, como desmotivación escolar, maltrato físico y psicológico, consumo de sustancias psicoactivas, entre otras, siendo recurrentes en los informes anuales en las ocho regiones de policía.

Según este informe, se presentaron 2.229 casos de riesgo psicosocial en los 22 colegios durante el 2018; es decir, un incremento del $50,5 \%$ con respecto a las cifras del 2017 , como se muestra en la tabla I.

Tabla I. Consolidado de riesgos psicosociales de los estudiantes de los colegios de la Policía Nacional 2018

\begin{tabular}{|lc|}
\hline \multicolumn{1}{|c}{ Tipología de riesgos } & Casos reportados \\
\hline Problemas de aprendizaje & $* 534$ \\
\hline Agresión física/verbal & $* 371$ \\
\hline Manejo del duelo & $* 100$ \\
\hline Agresividad & $* 198$ \\
\hline Baja autoestima & $* 206$ \\
\hline Desmotivación escolar & $* 523$ \\
\hline Depresión & 88 \\
\hline Deserción escolar & 30 \\
\hline Abuso sexual & 10 \\
\hline Maltrato psicológico & 20 \\
\hline Maltrato físico & 27 \\
\hline Sospecha de consumo & 42 \\
\hline Posesión y consumo & 12 \\
\hline Intento de suicidio & 4 \\
\hline Ideación suicida & 38 \\
\hline Estudiantes embarazadas & 4 \\
\hline Consumo de cigarrillo & 15 \\
\hline Consumo de bebidas alcohólicas & 7 \\
\hline \multicolumn{1}{|c|}{ Total } & 429 \\
\hline
\end{tabular}

Fuente: Informe de Riesgos Psicosociales 2018 del Observatorio de la Familia Policial. El asterisco $(*)$, indica las cifras más altas.

Con la tabla I se evidencia que los riesgos psicosociales con mayores índices son: problemas de aprendizaje (534 casos), seguido de agresión verbal/física (37I casos), manejo del duelo (100 casos), agresividad (I 98 casos), baja autoestima (206 casos) y desmotivación escolar (523 casos). Sin embargo, dentro de los riesgos identificados, aunque con menor presentación, también está el consumo de sustancias psicoactivas, maltrato físico y psicológico e intento suicida, solo por mencionar algunos de estos.

Teniendo en cuenta que las afectaciones al núcleo familiar repercuten gravemente en los estudiantes, como lo mencionan diferentes autores y teorías, también se evaluaron 
los riesgos psicosociales en los padres de familia, que se muestran en la tabla 2.

Tabla 2. Consolidado de riesgos psicosociales de los padres de familia, colegios, 2018

\begin{tabular}{|lc|}
\hline \multicolumn{1}{|c}{ Tipología de riesgos } & Casos reportados \\
\hline Orientación de tareas & $* 152$ \\
\hline Infidelidad & 27 \\
\hline Depresión & 15 \\
\hline Manejo de duelo & 47 \\
\hline Pautas de crianza deficientes & $* 790$ \\
\hline Ausencia de responsabilidad & $* 121$ \\
\hline Separación de los padres & $* 116$ \\
\hline Manejo inadecuado de los recursos & 40 \\
\hline Padres separados & $* 157$ \\
\hline Abuso sexual & 2 \\
\hline Consumo de sustancias & 2 \\
\hline Padres secuestrados & 0 \\
\hline Ideación suicida & 2 \\
\hline Intentos suicidas & 0 \\
\hline Abuso de bebidas embriagantes & 2 \\
\hline Violencia intrafamiliar & 16 \\
\hline \multicolumn{1}{|c|}{ Total } & 1.489 \\
\hline
\end{tabular}

Fuente: Informe de Riesgos Psicosociales 2018 del Observatorio de la Familia Policial. El asterisco $(*)$, indica las cifras más altas.

La tabla 2 evidencia 1.489 casos de riesgo psicosocial correspondientes al 2018, que al compararse con los 800 casos presentados en el 2017, se observa un incremento significativo. Para el caso de los padres de familia, los problemas de mayor presentación fueron las deficiencias en las pautas de crianza, la separación de las familias, las dificultades en la orientación de tareas de los niños y la ausencia de responsabilidades respecto a la crianza.

Teniendo en cuenta las problemáticas psicosociales que afectan a los estudiantes de los colegios de la Policía Nacional, en las ocho regiones de policía, se torna importante conocer cómo estos problemas vinculados a entornos familiares, escolares y del desarrollo individual de los estudiantes, afectan su desempeño académico. Se espera que al identificar estos riesgos, se diseñen estrategias de afrontamiento y mejora de los factores desfavorables; pretendiendo así que se evidencie una disminución en los índices de bajo desempeño académico y de problemáticas psicosociales en los estudiantes en los próximos años. Considerando lo anterior, surge la necesidad de responder: ¿existe relación entre los riesgos psicosociales y el desempeño académico en estudiantes de tres colegios en la ciudad de Bogotá?

\section{Marco teórico}

Los riesgos psicosociales surgen como un constructo teórico de difícil definición, si se toma en cuenta que existen diversas explicaciones desde la perspectiva de diferentes autores; sin embargo, para este estudio se toma como referencia la definición propuesta por la "Guía para una intervención integral en prevención de los riesgos psicosociales en niños, niñas y adolescentes" (20I2), que la define así:

El conjunto de circunstancias, hechos y factores personales, familiares o sociales, relacionados con una problemática determinada (abandono, maltrato, violencia, baja escolaridad, explotación económica, vínculos familiares precarios, necesidades básicas insatisfechas, entre otros) que aumentan la probabilidad de que un sujeto inicie o se mantenga en situaciones críticas que afectan a su desarrollo integral (deserción escolar, bajo desempeño académico, timidez o agresividad, entre otros), o que sea víctima o autor de la violencia u otras situaciones mencionadas afectando sus derechos esenciales (p. 12).

Siendo más específicos, los riesgos psicosociales pueden explicarse como la relación existente entre aspectos familiares, sociales y escolares, entre otras, $y$ las vulnerabilidades de cada individuo, haciendo referencia a los estilos de comunicación, resolución de conflictos y tolerancia a la frustración, por nombrar algunos.

De acuerdo con esta definición, los riesgos psicosociales pueden aumentar la probabilidad que se presenten o promuevan comportamientos disfuncionales en el desarrollo integral del ciclo vital de los individuos, especialmente en una etapa crítica de desarrollo y transición como lo es la adolescencia, que desde la perspectiva de autores como Coleman y Hendry (2003), Frías, López y Díaz (2003) y Saavedra (2004), se encuentra marcada por cambios de carácter físico y psicológico, que lleva a incursionar en diferentes crisis que modifican sus comportamientos y la percepción del mundo que los rodea; así como la manera de afrontar las situaciones problemáticas que se presentan de manera cotidiana, afectando no solo las relaciones y dinámicas familiares, sino también las de sus entornos próximos como el colegio, en donde la reunión de 
diferentes factores externos, con la reunión de características intrínsecas cambiantes, contribuye para que los adolescentes manifiesten problemas escolares de diferentes tipos.

Para autores como Deza (2015) y entidades como el Sistema para el Desarrollo Integral de la Familia del Estado de Jalisco, existen varios factores de riesgo psicosocial, los que pueden clasificarse así:

- Factores individuales: hacen referencia a rasgos personales que en ocasiones generan algunas dificultades en la relación con el entorno, como problemas de aprendizaje, baja capacidad para resolver problemas y conflictos.

- Factores familiares: estos se encuentran conformados por problemas, como la baja cohesión del núcleo familiar, la pobreza como minimizador de posibilidades de desarrollo; factores relacionados a la aceptación o rechazo de los pares en relación con la pertenencia a grupos.

- Factores escolares: que siendo una de las instituciones más relevantes para los procesos de socialización, incide de manera determinante en el desarrollo integral de los niños y sus patrones de comportamiento.

- Factores sociocomunitarios: referentes a los entornos comunitarios en los que se desenvuelven, crecen y se desarrollan la niñez y la adolescencia de cada individuo, tomado como factor relacional para un futuro desarrollo en valores y costumbres.

- Factores socioculturales: los estereotipos imperantes a nivel cultural manejados y ejercidos por personas e instituciones, determinan actitudes y posturas, lo que deriva en muchas ocasiones en estigmatizaciones hacia niños, niñas y adolescentes.

Son justamente los factores descritos líneas arriba, los que al estar en interacción con las características individuales en diferentes situaciones se convierten en factores protectores o de riesgo. Sobre los factores de riesgo, Deza (2015) define una condición de riesgo como

la interacción de factores externos (influencia de pares, familia, escuela, comunidad y cultura) con vulnerabilidades individuales (características cognitivas, capacidad de resolución de conflictos, tolerancia a la frustración, etc.) que sensibilizan a ciertos individuos que se encuentran más expuestos ante estos (p. 232).

En cuanto a los factores protectores, Bonilla y Marcilia (20I5) mencionan que estos permiten el desarrollo de una autoestima positiva, favorecen el desarrollo de destrezas sociales, cognitivas y emocionales, propician el afrontamiento de problemas y la toma decisiones, aportan a la resolución de situaciones de riesgo de manera exitosa, además de disminuir el efecto negativo de los riesgos psicosociales y actúan como barrera de estos, que pueden llegar a causar afectaciones importantes en todas las esferas de un individuo. Donas (200I), citado por Bonilla y Marcilia (2015), define los factores protectores como

aquellas características que se pueden detectar, potencializar y desarrollar en un sujeto (...) favoreciendo el desarrollo humano, el mantenimiento o la recuperación de la salud en el sentido amplio de lo biológico, psicológico y social; y que además pueden contrarrestar los posibles efectos de los factores de riesgo, de las conductas de riesgo y, por lo tanto, reducir la vulnerabilidad, ya sea general o específica (p. 26).

Además, algunos factores personales se convierten en factores protectores como ser empático, tener altos niveles de autoestima, motivación al cumplimiento de metas, autonomía e independencia, capacidades de resolución de problemas (Medina \& Carvalho, 20 I0). Otros más relacionados al aspecto actitudinal, es decir, a las actitudes que posee cada individuo, son la alta valoración de sí mismo, positivismo ante las situaciones problemáticas y autocontrol, desarrollo de habilidades para la resolución de conflictos, regulación emocional y forma de relacionarse con pares $y$ adultos.

Para este mismo autor, no solo los factores personales se convierten en protectores, también están los factores familiares, sociales y escolares; en el factor familiar, se encuentran las relaciones positivas con los miembros del núcleo familiar, normas y pautas de conducta claras y fiables, unión entre los miembros de la familia y el cuidado mutuo.A su vez, el factor social hace referencia a actividades que crean responsabilidad social en los adolescentes y niños, como la participación en obras que beneficien a la comunidad y la identificación de modelos a seguir diferentes a los padres. Finalmente, en los factores relacionados con el contexto escolar, los que se tornan importantes en cuanto al tiempo y las relaciones que se desarrollan en los colegios, se encuentran el clima positivo y favorecedor que rodea al niño y la adaptación o las habilidades de integración de este, las que minimizan el impacto de los riesgos psicosociales (Bonilla \& Marcilia, 20I5).

Donas (200I) menciona otros factores protectores: los de amplio espectro y los específicos. En los primeros está la familia como red de apoyo, la habilidad de comunica- 
ción interpersonal; un nivel alto de autoestima; un locus de control interno; permanecer en el sistema educativo formal y altos niveles de resiliencia. Respecto a los factores protectores específicos menciona: los que reducen o evitan los comportamientos que generen daño para el individuo, como los que implican responsabilidad y autocuidado, así como evitar fumar, tener relaciones sexuales con protección ante enfermedades de transmisión sexual y embarazos no deseados, evitar el consumo y el abuso de sustancias psicoactivas y embriagantes, entre otras.

Por tanto, puede inferirse que cada individuo se relaciona con diferentes contextos (familiar, escolar, social, etc.), los que en conjunto con sus características individuales resultan en diferentes patrones de comportamiento, donde dependiendo de estos contextos y características, pueden convertirse en factores de riesgo o en factores protectores. Pues se entiende que cuando los factores de riesgo se minimizan, se convierten en factores de entorno protector; por el contrario, cuando estos riesgos se intensifican, se aumenta la probabilidad de ocurrencia de comportamientos desadaptativos y peligrosos para una persona.

Es por consiguiente, importante desarrollar estudios en el ámbito de riesgo psicosocial con poblaciones infantiles y adolescentes, que trasciendan no solo a la identificación de los mismos, donde se expone esta población, sino que también permitan determinar aspectos relevantes como la relación con otros problemas, la influencia y afectación en diferentes entornos y esferas como la familiar, interpersonal y académica, entre otros; específicamente, caracterizando estos estudios en poblaciones particulares que permitan desarrollar estrategias de intervención y prevención, ajustándose a sus características y conllevando una mayor efectividad.

El desempeño académico es una de las variables con las que se ha relacionado el riesgo psicosocial. Autores como Hernández, Coronado,Araújo y Cerezo (2008) definen el desempeño académico como "el grado de logro de los objetivos establecidos en los programas escolares. Este indicador es de tipo multidimensional (...) ya que involucra variables de orden cognitivo, volitivo y emocional" (p. 15). El desempeño académico entonces se considera como una manera medible para cuantificar la suma del trabajo obtenido por un individuo en un área específica del aprendizaje.

Un ejemplo de estos estudios que relacionan los riesgos psicosociales y rendimiento académico o desempeño escolar es desarrollado por Gutiérrez, Camacho y Martínez (2007), quienes buscaron identificar la relación entre rendimiento escolar, autoestima y los riesgos en la funcionalidad familiar de adolescentes escolarizados de 10 a 17 años de edad. Encontraron que existe una relación directa entre el desempeño académico y la autoestima, donde a mayor autoestima se alcanza mayor rendimiento escolar, mientras que la menor autoestima conlleva un menor rendimiento. Es por esto por lo que los riesgos en el funcionamiento familiar cuentan con un factor que afecta altamente la autoestima, según este estudio.

Con estas mismas variables se muestra el estudio llevado a cabo por Cardona (2017); en este no solo se caracterizan los riesgos, sino que también se desarrolla un plan de trabajo con una serie de estrategias didácticas y de intervención para minimizar el impacto de los riesgos psicosociales en el desempeño académico de los estudiantes en condiciones de vulnerabilidad y contextos de violencia y desplazamiento.

Otro de los estudios que vincula la variable de riesgo psicosocial, es el que se llevó a cabo entre la coordinación con los centros escolares de referencia y el Programa Integral Nacional para el Bienestar Social (INABIF-MIMDES) en Perú en el 2012; y buscó identificar el nivel de riesgo psicosocial en los estudiantes entre 3 y 17 años de las instituciones educativas de Piura, Cajamarca e lquitos, contó con la participación de 155 niños, niñas y adolescentes, 69 padres y 153 docentes. Allí se aplicó el instrumento de identificación del riesgo psicosocial en niños, niñas y adolescentes, también se realizaron talleres, grupos focales, y se encontró que los factores de riesgos con niveles más altos son el maltrato físico del padre, la ausencia de los padres y la agresividad con pares o hermanos. Otros factores que se muestran como relevantes, aunque con menor nivel, son el trabajo infantil, las limitaciones para continuar con el estudio, donde recalcan los problemas en el hogar como un factor que aumenta el nivel del riesgo, las conductas inapropiadas y el inicio temprano de la maternidad.

Otro ejemplo en Latinoamérica, es la investigación desarrollada en Ciudad de México, con 32I estudiantes de bachillerato y licenciatura, por Pulido et al. (20I3), quienes evaluaron once riesgos psicosociales como predictores de consumo de tabaco, drogas, alcohol y conducta sexual de riesgo, por medio de un cuestionario aplicado. Los resultados muestran que los factores de riesgo como el estrés académico y los centros de distribución cercanos, 
promueven el consumo de tabaco y alcohol, mientras que los factores como distribuidores cercanos contribuyen al consumo de drogas; respecto a las conductas sexuales de riesgo, es la imitación social la que promueve su aparición. Este estudio también identificó algunos factores protectores, entre los cuales están la religiosidad, la asertividad y percepción de riesgo.

Refiriéndose específicamente al contexto colombiano, también es posible encontrar investigaciones que identifican los factores de riesgo psicosocial en estudiantes adolescentes, como la que proponen Giraldo y Lotero (2018), que aplicaron una encuesta a 128 alumnos de los grados noveno, décimo y once de la institución educativa Evaristo García, para identificar los factores de riesgo psicosocial en los ámbitos familiar, escolar y comunitario. Como resultado de estudios anteriores, los factores de riesgo con mayor relevancia encontrados son en el entorno personal, pensamientos suicidas y depresión; en el entorno escolar, constante robo de elementos personales, porte de armas, conflictos o peleas, embarazos no deseados y contaminación ambiental. A su vez, en el entorno familiar se postulan como riesgos psicosociales: conflictos familiares, falta de comunicación y desconfianza. Para finalizar, mencionan que los riesgos encontrados en el entorno social son el expendio y consumo de drogas, la presencia de pandillas, los robos frecuentes, las zonas de alto riesgo de inundación y un evidente porcentaje de contaminación ambiental.

Finalmente, se menciona, en algunos estudios, la relación del riesgo psicosocial con otras variables en niños, niñas y adolescentes escolares, como las habilidades cognitivas propuestas por Navarro y Navarro (2017), que describen las características de la habilidad cognitiva según la prevalencia de los factores de riesgo psicosocial presentes en niños de básica primaria; o relacionado con factores como el riesgo psicosocial y la resiliencia en adolescentes (Cardozo \& Alderete, 2009).

\section{Metodología}

\section{Tipo de investigación y diseño}

Este estudio se realiza desde un enfoque cuantitativo centrado en las medidas numéricas que "se fundamenta y utiliza la observación del proceso en forma de recolección de datos y los analiza para llegar a responder las preguntas que se plantean en un inicio de la investigación"
(Cabezas, Andrade \& Torres, 20I8, p. 66) y un diseño no experimental-correlacional, que como lo menciona Sousa, Driessnack y Costa (2007), busca "describir variables y las relaciones que se dan de forma natural entre las mismas" (p. 3). La recolección de datos es trasversal, pues pretende obtener la información en un único periodo de tiempo.

\section{Participantes}

Se realizó un muestreo censal definido por Ramírez, citado en Quispe (2017), como "aquella donde todas las unidades de investigación son consideradas como muestra" (p. 77), puesto que se contaba con la disposición del $100 \%$ de la población, conformada por 650 estudiantes de tres instituciones educativas pertenecientes a la Policía Nacional, en Bogotá, con edades entre I 3 y I5 años de los grados octavo, noveno y décimo.

La participación fue voluntaria y cada estudiante firmó un asentimiento informado, adicional al consentimiento que firmaba el padre o representante legal para autorizar la participación. No se ofreció a los voluntarios ningún incentivo económico o de otro tipo por su participación y es importante aclarar que este estudio fue avalado por el comité de investigación de la Dirección de Bienestar Social de la Policía, así como por la Vicerrectoría de Investigación de la Dirección Nacional de Escuelas de la Policía Nacional de Colombia.

La edad mínima tanto para hombres como para mujeres era de 13 años, y la máxima de 15 , información que se utilizó como criterio de exclusión, así como la firma de los consentimientos y asentimientos (véase tabla 3). La población se distribuyó en un porcentaje de participación del $64 \%$ para las mujeres, y $36 \%$ para los hombres.

\section{Instrumentos o técnicas}

El Cuestionario para la Evaluación de Problemas en Adolescentes, desarrollado por Sica, Chirri, Favilli y Marchetti en el 20l ly adaptada al español por Santamaría (2016), está compuesto por 81 ítems que evalúan nueve escalas, algunas de ellas de índole clínico (depresión, ansiedad, insatisfacción corporal), pero también evalúan escalas de riesgo psicosocial, bienestar y autoestima, problemas familiares, interpersonales, abuso de sustancias psicoactivas e incertidumbre sobre el futuro. Estimando así un panorama general de los factores de riesgos asociados a los adolescentes entre 12 y 18 años, con un tiempo de aplicación entre 20 a 30 minutos, ya sea de forma grupal o individual. En la tabla 4 se muestra una breve definición de cada escala. 
Tabla 3. Conformación de la población

\begin{tabular}{|c|c|c|c|c|c|c|}
\hline \multicolumn{3}{|c|}{ Colegio I } & \multicolumn{2}{|c|}{ Colegio 2} & \multicolumn{2}{|c|}{ Colegio 3} \\
\hline Edad & Hombre & Mujer & Hombre & Mujer & Hombre & Mujer \\
\hline 13 & 11 & 20 & 30 & 30 & 33 & 34 \\
\hline 14 & 20 & 27 & 47 & 61 & 46 & 54 \\
\hline 15 & 12 & 39 & 44 & 45 & 47 & 50 \\
\hline Subtotal & 43 & 86 & 121 & 136 & 126 & 138 \\
\hline Total por colegio & \multicolumn{2}{|c|}{129} & \multicolumn{2}{|c|}{257} & \multicolumn{2}{|c|}{264} \\
\hline Total & \multicolumn{6}{|c|}{650} \\
\hline
\end{tabular}

Tabla 4. Escalas del Cuestionario para la Evaluación de Problemas en Adolescentes

Insatisfacción corporal

Ansiedad

Depresión

Abuso de sustancias

Problemas interpersonales

Problemas familiares

Incertidumbre sobre el futuro

Riesgo psicosocial

Autoestima y bienestar
Evalúa la presencia de insatisfacción con su aspecto físico y con su peso, que podría implicar conductas de alimentación potencialmente problemáticas (dietas muy estrictas, rechazo a la comida, atracones, etc.). Suele ir acompañada de problemas emocionales, malestar relacionado con la percepción corporal y sensibilidad interpersonal.

Evalúa la presencia de manifestaciones subjetivas de ansiedad, tensión y preocupación tanto cognitivo como emocional y fisiológico ("A menudo estoy tan nervioso o nerviosa que puedo notar cómo me late el corazón con fuerza").

Evalúa la presencia de manifestaciones subjetivas de tristeza, melancolía, anhedonia o desesperanza ("Da igual lo que haga, las cosas no van a mejorar").

Evalúa la presencia de abuso de sustancias y el grado en que puede condicionar el funcionamiento del adolescente en diferentes contextos de su vida. Los ítems de esta escala hacen referencia a las consecuencias negativas del uso de sustancias, de tal manera que las preguntas no susciten demasiada resistencia por parte de los adolescentes.

Evalúa la presencia de insatisfacción y dificultades tanto a la hora de relacionarse con los demás como de gestionar los conflictos. Incluye la percepción de sus relaciones con los demás como decepcionantes, conflictivas y frustrantes, lo que le lleva a un cierto distanciamiento u oposición.

Los ítems de esta escala hacen referencia a conflictos y experiencias de incomprensión familiares, así como al nivel en que estos problemas afectan a su funcionamiento y comportamiento.

Los ítems de esta escala se refieren a la indecisión y preocupación sobre su futuro escolar, académico o laboral que afectan al bienestar del adolescente y que requiere de intervención y orientación.

Evalúa el grado en que la persona tiene dificultades para adaptarse a las reglas sociales, para saber controlar su comportamiento e inhibir las conductas que impliquen un riesgo para su persona y para los demás, ya sea por impulsividad o desinterés.

Proporciona una medida global y sintética del nivel de bienestar del adolescente. Evalúa su grado de satisfacción consigo mismo, con su entorno y con su estado.

Fuente: recuperado de TEA Ediciones (http://www.web.teaediciones.com/Ejemplos/QPAD-Presentaci\%C3\%B3n.pdf)

Es importante mencionar que para dar solución a los objetivos de este estudio, se tomó en cuenta únicamente los resultados de la escala de riesgo psicosocial, la cual se analizó por medio del software estadístico SPSS STATISTICS versión 26 .

\section{Procedimiento}

Una vez el proyecto de investigación contó con la aprobación del director de la Dirección de Bienestar Social, el grupo de educación y de los directivos de los planteles educativos solicitó la colaboración del grupo de profesionales en psicología pertenecientes a los tres colegios, para realizar la aplicación de forma simultánea del Cuestionario para la Evaluación de Problemas en Adolescentes.
A estos profesionales, se les capacitó en la aplicación de la prueba de manera colectiva. Cada estudiante participante de esta investigación firmó el asentimiento informado y sus padres o tutores legales el respectivo consentimiento informado, que explicaba las consideraciones generales y éticas de la participación, aceptando además su participación voluntaria.

La aplicación de los cuestionarios se realizó de manera grupal con la participación de 20 a 35 adolescentes. Para llevarla a cabo, el profesional en psicología leyó cada una de las $8 \mathrm{I}$ preguntas, mientras que el estudiante, en la hoja marcaba la respuesta con la que se sentía más identificado: en una escala Likert, siendo I, no me describe para nada 
y 4, describe bien mi situación u opinión. Es importante mencionar que la aplicación de este cuestionario se acompañó del desarrollo de un taller de aproximadamente 30 minutos, sobre temas como proyecto de vida, autoestima, autorregulación, entre otros, dependiendo de la necesidad de cada curso.

Posteriormente, al obtener la puntuación de todos los participantes, se utilizó la plataforma TEA.EDICIONES para emitir el perfil individual de los participantes. Para continuar con la consolidación de las notas que representa el desempeño académico de todos los estudiantes, el área de coordinación académica de cada colegio emitió los informes académicos de los tres primeros periodos de los estudiantes. Las calificaciones se encuentran representadas así: (B-) con la nota más baja, seguido de básico (B), desempeño alto $(A)$ y superior $(S)$, para representar la nota más alta; como última fase, se hizo el análisis estadístico de los datos con el software Excel y SPSS Statistics versión 26 , con el cual fue posible obtener los datos descriptivos y correlacionales.

\section{Análisis de resultados}

Una vez se obtuvieron los 650 perfiles con los resultados en percentiles de las nueve escalas tanto clínicas como de autoestima y bienestar y riesgo psicosocial, se determinó cuántos estudiantes puntuaron con un percentil > a 90 , lo que refiere un malestar clínico significativo, exceptuando la escala de autoestima y bienestar, donde son los puntajes < a 20 los que indican un malestar y problemas clínicos destacados, como se muestra la figura $I$.

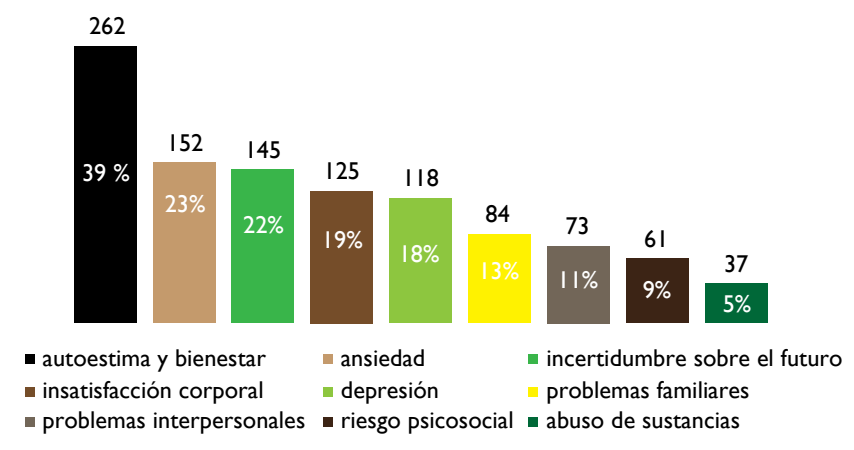

Figura I. Consolidado general de resultados del Cuestionario para la Evaluación de Problemas en Adolescentes, en los tres colegios

Nota. Unidad de medida, número de casos. La sumatoria del resultado porcentual no es $100 \%$, teniendo en cuenta que cada perfil puede presentar puntajes mayores al percentil 90 en más de una escala.

En la figura I se observa el consolidado total de la puntuación que representan malestar y problemas clínicos destacados (percentil > 90) en las nueve escalas que evalúa el cuestio- nario. Allí se encuentra, en primer lugar, la escala de autoestima y bienestar con 265 casos reportados, siendo el $39 \%$ de todas las problemáticas de la muestra; seguido de la escala de ansiedad con 152 casos y $23 \%$; después se encuentra la escala de insatisfacción corporal y depresión con el $19 \%$ y $18 \%$, respectivamente; con el $13 \%$ y $11 \%$ se encuentran las escalas de problemas familiares y problemas interpersonales, para terminar con las escalas de riesgo psicosocial y abuso de sustancias psicoactivas con el $9 \%$ y $5 \%$, respectivamente.

Con el fin de informar a los padres, estudiantes y directivos sobre los resultados del cuestionario aplicado y activar las rutas de remisión a entidades prestadoras de salud, se categorizaron los riesgos psicosociales en cuatro grupos, como se muestra en la tabla 5 .

Tabla 5. Consolidado de la categorización de escalas

\begin{tabular}{|c|c|c|c|}
\hline Número de escalas & Categorías & $\mathbf{N}$ & Porcentajes \\
\hline 0 & 0 & 237 & $37 \%$ \\
\hline I a 2 & 1 & 253 & $39 \%$ \\
\hline 3 a 4 & 2 & 110 & $17 \%$ \\
\hline 5 a 6 & 3 & 35 & $5 \%$ \\
\hline 7 a 8 & 4 & 15 & $2 \%$ \\
\hline \multicolumn{2}{|l|}{ Total } & 650 & $100 \%$ \\
\hline
\end{tabular}

Según la tabla 5 , se organizaron los casos en cuatro categorías de manera ascendente, para finalmente identificar que la mayoría de los casos se centraron en la categoría 0 : estudiantes que no presentaron puntajes con relevancia clínica en ninguna de las escalas, 237 casos (37\%). En la categoría I se sitúan a los estudiantes que presentaron un resultado relevante ( $>90$ percentil) en una y dos de las escalas evaluadas, obteniendo 253 casos (39\%); II0 casos están agrupados en la categoría 3, que presentan entre 5 y 6 escalas, con un total de 35 casos identificados (5\%); y, en último lugar, se sitúa la categoría 4 que agrupa a quienes tienen puntajes significativos en 7 y 8 de las escalas en la categoría 4 con un total de 15 casos (2\%).

En relación con la variable sexo, como se muestra en la figura 2, la participación fue mayor en las mujeres con $64 \%$ que la de los hombres con $36 \%$. También se buscó conocer, por medio de una prueba de diferencia de medias, cuál de los dos sexos tenía mayor riesgo psicosocial; los resultados de la tabla 6 evidencian que las mujeres tienen mayor riesgo psicosocial con un valor del 0,62 , en comparación con los hombres, que tienen un valor de 0,33. 


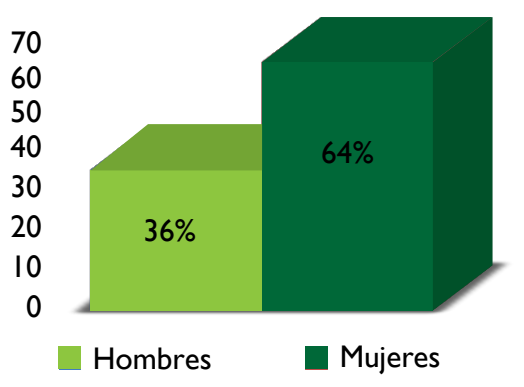

Figura 2. Sexo respecto al riesgo psicosocial

Tabla 6. Comparación de medias para la variable sexo

\begin{tabular}{ccc} 
Sexo & Media & Desviación \\
Mujeres &, 62 &, 653 \\
Hombres &, 33 &, 558 \\
\hline
\end{tabular}

En cuanto a los análisis de correlación entre el desempeño académico y el riesgo psicosocial, una vez se obtuvieron las notas (B-, B,A, S) entregadas por cada colegio, se transformaron estas a escala numérica de forma ascendente ( $I$, 2, 3, 4), donde I equivale a (B-), 2 equivale a (B) y así sucesivamente; para con este dato, por medio de la prueba Bootstrap o de remuestreo, que no requiere una distribución normal de las variables, identificar los coeficientes de correlación.

Tabla 7. Coeficientes de correlación entre las escalas de la prueba Q-PAD y la variable desempeño académico

\begin{tabular}{lcc}
\multicolumn{3}{c}{ Desempeño académico } \\
Escalas de la prueba Q-PAD & $\begin{array}{c}\text { Correlación } \\
\text { de Pearson }\end{array}$ & $\begin{array}{c}\text { Valor } \boldsymbol{p} \\
\text { (unilateral) }\end{array}$ \\
\hline Insatisfacción corporal &, $075^{*}$ &, 028 \\
\hline Ansiedad &, 030 &, 222 \\
\hline Depresión &,- 037 &, 173 \\
\hline Abuso de sustancias &,$- 147^{* *}$ &, 000 \\
\hline Problemas interpersonales &,- 014 &, 365 \\
\hline Problemas familiares &,- 058 &, 070 \\
\hline Incertidumbre sobre el futuro &, $097^{* *}$ &, 007 \\
\hline Riesgo psicosocial &,$- 099^{* *}$ &, 006 \\
\hline Autoestima y bienestar &,$- 097^{* *}$ &, 007 \\
\hline Nota: &
\end{tabular}

Nota: $* *$ la correlación es significativa al nivel $0,0 \mathrm{l}, *$ la correlación es significativa al nivel 0,05 .

Según la tabla 7, se observa que si bien existe una correlación entre el desempeño académico y las escalas que puntúan con una significancia menor que $(p<0,05)$, las que para este caso son insatisfacción corporal $(r=, 075)$; abuso de sustancias psicoactivas $(r=-, 147)$; incertidumbre sobre el futuro $(r=, 097)$; autoestima y bienestar $(r=-, 097)$ y riesgo psicosocial $(r=, 099)$, esta última con el mayor puntaje, estos valores representan una correlación muy baja y poco significativa (en un rango entre $0,0 \mathrm{l}$ a 0,3 ), e indican que la variable desempeño académico, no evidencia una correlación significativa ni con el riesgo psicosocial, ni con ninguna otra de las escalas del cuestionario.

Para la variable de estudio, tipología de familia, tanto para aquellos adolescentes que puntuaron significativamente en una escala o más, como para aquellos que no puntuaron en ninguna de las escalas, es la familia nuclear, la manera más común de conformación familiar, seguido de la familia monoparental, para quienes puntuaron de manera significativa en alguna escala y la familia extensa, para quienes no puntuaron en ninguna de las escalas (véase figura 3 ).

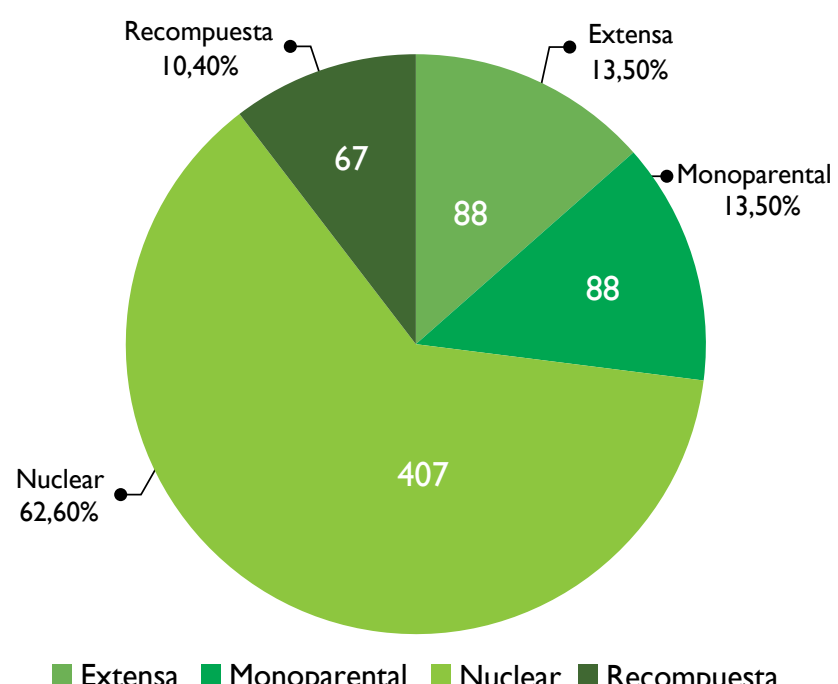

Figura 3. Descriptivos de la variable tipología de familia

En cuanto a esta misma variable y el desempeño académico, también se realizó una prueba de rangos de KruskalWallis para identificar si existía una diferencia entre la variable desempeño académico y la tipología familiar. Los resultados se muestran en la tabla 8 .

Tabla 8. Prueba de rangos de Kruskal-Wallis

\begin{tabular}{cccc}
\hline & Tipología de familia & N & Rango promedio \\
\hline & Extensa & 88 & 286,56 \\
\hline \multirow{2}{*}{$\begin{array}{c}\text { Desempeño } \\
\text { académico }\end{array}$} & Nuclear & 48 & 330,93 \\
\cline { 2 - 4 } & Recompuesta & 67 & 337,94 \\
\hline & Total & 650 & 293,96 \\
\hline
\end{tabular}


Según los datos del rango promedio, es posible afirmar que los estudiantes provenientes de una familia extensa $(286,56)$ evidencian un bajo desempeño académico, en comparación con los otros tipos de conformación familiar. Por el contrario, los estudiantes provenientes de una familia nuclear $(337,94)$, presentan un nivel alto de desempeño académico en comparación con los demás tipos de familia.

Tabla 9. Descriptivos sobre la variable tipología de familia

\begin{tabular}{c|ccc}
\hline Escala & Tipología de familia & N & Media \\
\hline & Extensa & 88 & 53,74 \\
& Monoparental & 88 & 59,68 \\
\hline \multirow{2}{*}{ Riesgo psicosocial } & Nuclear & 407 & 56,39 \\
\cline { 2 - 4 } & Recompuesta & 67 & 66,81 \\
\hline & Total & 650 & \\
\hline
\end{tabular}

La tabla 9, a su vez, indica que los resultados de la media en la familia extensa $(53,74)$, presenta el menor riesgo psicosocial entre los demás tipos de familia estudiados. En la muestra de aquellos adolescentes que pertenecen a la familia recompuesta, evidencian un mayor riesgo psicosocial que aquellos que provienen de otros tipos de familia.

\section{Discusión}

Este trabajo estudió el riesgo psicosocial y el desempeño académico en un grupo de 650 adolescentes entre los 13 y 15 años de edad, pertenecientes a los colegios de la Policía Nacional en la ciudad de Bogotá, con el fin de determinar si efectivamente estos factores descritos se encuentran relacionados y de qué forma; se analizó además, la influencia con otras variables como el sexo y la tipología o conformación familiar.

Respecto al desempeño académico, los resultados evidenciaron que aunque existe una relación entre esta variable y las escalas de riesgo psicosocial insatisfacción corporal, abuso de sustancias psicoactivas, incertidumbre sobre el futuro y la autoestima y el bienestar, de forma contraria a la hipótesis planteada en este estudio, esta no es lo suficientemente significativa para afirmar que el riesgo psicosocial incide en el desempeño académico u otras de estas escalas analizadas.

Sin embargo, este estudio encontró hallazgos significativos en la tipología de familia o conformación familiar de los estudiantes, donde se evidenció que la familia nuclear es la más común, tanto en adolescentes que puntuaron significativamente en la escala de riesgo psicosocial, como los que no puntuaron; $y$ son, justamente, los estudiantes que pertenecen a una familia nuclear, los que revelan el desempeño académico más alto en comparación con los otros tipos de familia. También se encontró que los estudiantes que provienen de una familia extensa tienen un desempeño académico más bajo, que aquellos que pertenecen a los demás tipos de familia, aunque son estos mismos los que presentan el menor riesgo psicosocial; sin embargo, respecto al análisis de los que no presentaron ninguna escala clínica, fue este tipo de familia la que prevaleció, por el contrario a quienes pertenecen a la familia recompuesta que representan el mayor riesgo evidenciado. Cabe resaltar que el tipo de familia monoparental fue el que se asoció a aquellos estudiantes que presentaron al menos una escala clínica o más.

La prevalencia del riesgo psicosocial entre hombres y mujeres, también fue una variable analizada; se encontró que son, justamente, estas últimas quienes presentan un mayor riesgo psicosocial, en comparación con los hombres y es justo allí donde los factores protectores mitigan los efectos negativos que estos riesgos tienen, de forma especial en contextos de pobreza

donde la educación de las niñas, hace que el embarazo se retrase hasta siete años por término medio, contribuye a que se eviten ciertas enfermedades como el sida, les capacita para empleos más cualificados y reducen la dependencia de los varones (Uriarte, 2006, p. 15).

Es importante mencionar que los resultados de este trabajo se consideran relevantes, debido a la escasez de estudios realizados en población adolescente que pertenece a contextos educativos en la Policía Nacional, y que evalúen las problemáticas relacionadas con los riesgos psicosociales, a los que se ven expuestos los estudiantes, y que analicen, además, variables como la conformación familiar y cómo afectan estos el entorno escolar, y que -como ya se mencionó- actúe como un factor protector que minimice los efectos negativos, de los riesgos psicosociales. Se espera que, en estudios posteriores, sea posible ampliar los análisis realizados sobre las demás escalas que evalúa el cuestionario de problemas en adolescentes Q-PAD, para así descubrir su incidencia en esta población.

Finalmente, se identificó, con una limitación en este estudio, la dificultad para realizar la aplicación a un grupo de estudiantes tan grande de forma simultánea, sin descuidar 
el control de las variables externas que pudieran afectar; por este motivo, la aplicación se hizo en diferentes momentos para cada colegio, $y$ así aumentar el control de las condiciones de aplicación de la prueba.

\section{Conclusiones}

Los hallazgos de este estudio en población con características tan particulares como lo son los adolescentes de colegios de la Policía Nacional, permiten concluir qué tipología o conformación familiar de los adolescentes tienen un papel de gran importancia, no solo porque -como se mencionó en el apartado de marco teórico- se desempeña como un factor protector ante los riesgos psicosociales a los que se ven expuestos los adolescentes, sino que también estos parecen relacionarse con el desempeño académico de los estudiantes.

Según lo anterior, deben aunarse esfuerzos en la comunidad académica, constituida por padres, docentes, directivos y orientadores, con el objetivo de identificar y desarrollar estrategias claras, específicas para las características de esta población y enfocadas en la disminución del impacto negativo que ejercen los riesgos psicosociales en todos los adolescentes $y$, de forma especial, en aquellos identificados con mayor riesgo psicosocial (mujeres provenientes de familias extensas), que brinden herramientas a cada uno de los actores involucrados en el contexto educativo para el afrontamiento de los riesgos psicosociales $y$, a su vez, fomenten unos adultos sanos y estables emocionalmente.

Para finalizar, se recomienda apostar por el fortalecimiento y promoción de los factores protectores mencionados en este estudio, desde diferentes contextos y, en especial, desde el ámbito familiar para promover espacios positivos y afectivos que redunden en el bienestar y en la salud de los hijos; así como la importancia del contexto educativo, ya que se ha identificado que incide en la disminución del impacto negativo de los riesgos psicosociales. Pues, como afirman diferentes autores, al ser un ambiente "positivo, orientador, con normas y valores claros; modelos sociales que valoren el enfrentamiento positivo de los problemas; equilibrio entre responsabilidades sociales y expectativas" (Cid-Monckton \& Pedrão, 20ll, p. 4), los factores protectores favorecen el desarrollo de las personas y son capaces de "reducir los efectos de circunstancias desfavorables" y "sobreponerse a la adversidad, crecer, y desarrollarse adecuadamente, llegando a madurar como seres adultos competentes", además de "evitar los daños permanentes en la persona (...) transformando una situación negativa en un factor de superación" (Solórzano, Gaitán, Uribe, Castro \& Llanes, 2007, p. 4).

\section{Financiación}

Proyecto financiado por la Dirección de Bienestar Social de la Policía Nacional. Observatorio de la Familia Policial.

\section{Referencias}

Bonilla, S. M., \& Marcilia,Y. (20I5). Factores protectores que favorecen la realización de proyecto de vida en adolescentes en riesgo psicosocial de la Institución Juventud con una Misión (tesis de especialización). Caldas, Antioquia, Colombia: Corporación Universitaria Lasallista.

Cabezas, E.,Andrade, D., \& Torres, J. (20I8). Introducción a la metodología de la investigación científica. Ecuador: Universidad de las Fuerzas Armadas ESPE. Recuperado de htpp//www. repositorio.espe.edu.ec.

Cardona,T. M. (2017). Riesgos psicosociales que afectan el aprendizaje de los estudiantes (tesis). Bello, Antioquia, Colombia: Universidad San Buenaventura.

Cardozo, G., \& Alderete, A. M. (2009). Adolescentes en riesgo psicosocial y resiliencia. Psicología desde el Caribe, 23, I48182.

Centros escolares de referencia. Programa Integral Nacional para el Bienestar Social (INABIF-MIMDES). (2012). Indicadores para la identificación del riesgo psicosocial de estudiantes: una mirada a la niñez en riesgo psicosocial.Lima, Perú. Recuperado de https://www.fad.es/wp content/uploads/2019/07/ Investigacion_IX.pdf.

Cid-Monckton, P., \& Pedrão, L. J. (20I I). Factores familiares protectores y de riesgo relacionados al consumo de drogas en adolescentes. Revista Latino-Americana de Enfermagem, 19, 738-745.

Coleman, J., \& Hendry, L. B. (2003). Psicología del adolescente. Madrid: Ediciones Morata, S.L.

Deza, V. S. (2015). Factores de riesgo y protección en niños y adolescentes en situación de pobreza de instituciones educativas en Villa El Salvador. Revista Avances en Psicología, 23(2), 23I-240. https://doi.org/I0.33539/avpsicol.20I5. v23n2. 165 
Donas, B. (200I). Adolescencia y juventud en América Latina. Costa Rica: Libro Universitario Regional.

Frías, M., López, A. E., \& Díaz, S. G. (2003). Predictores de la conducta antisocial juvenil: un modelo ecológico. Estudios de la Psicología, (8), I5-24.

Giraldo, D., \& Lotero, Y. (20I8). El riesgo psicosocial en los estudiantes adolescentes de la "Institución Educativa Evaristo García" de Santiago de Cali (tesis de pregrado). Cali, Colombia: Universidad Nacional Abierta y a Distancia - Unad.

Guía para una intervención integral en prevención de los riesgos psicosociales en niños, niñas y adolescentes. (2012). Recuperado de https://www.fad.es/ wp-content/uploads/2019/05/Gu\%C3\%ADa-para-unaintervenci\%C3\%B3n-integral-en-prevenci\%C3\%B3n-delos-riesgos-psicosociales-en-ni\%C3\%Blos-ni\%C3\%Blasy-adolescentes.pdf.

Gutiérrez, P., Camacho, N., \& Martínez, M. (2007). Autoestima, funcionalidad familiar y rendimiento escolar en adolescentes. Revista Originales, 39( I I), 597-660. https://doi. org/10.1157/13112196

Hernández, P. M., Coronado, Á. O.,Araújo, C.V., \& Cerezo, R. S. (2008). Desempeño académico de universitarios en relación con ansiedad escolar y auto-evaluación. Acta Colombiana de Psicología, I I (I), I3-23.

Medina, A. N., \& Carvalho, F. M. (2010). Factores protectores de las familias para prevenir el consumo de drogas en un municipio de Colombia. Revista Latino-Americana de Enfermagem, I8, 504-5। 2 .
Navarro, L., \& Navarro,Y. (20I7). Factores de riesgo psicosocial y habilidades cognitivas en niños de básica primarias (tesis de pregrado). Puerto Berrío, Antioquia, Colombia: Universidad de Antioquia.

Pulido, M., Cortés, L., Melo, S., Domínguez, Y., Pedraza, F., \& Pérez, A. (2013). Predictores de riesgos psicosociales en estudiantes de bachillerato y licenciatura. Revista Electrónica de Psicología Iztacala, I6(2), 504-532.

Quispe, R. (2017). Percepción en los periodistas de las emisoras de amplitud modulada sobre el periodismo tradicional y digital. Puno, 2016 (tesis de maestría de pregrado). Puno, Perú: Universidad Nacional del Altiplano.

Saavedra, R. M. (2004). Cómo entender a los adolescentes para educarlos mejor. México: Editorial Pax México.

Solórzano, N., Gaitán, P., Uribe, M., Castro, M. E., \& Llanes, J. (2007). Estudio de riesgo protección psicosocial entre los estudiantes de la Universidad Iberoamericana Ciudad de México. Liberaddictus, Cuadernos de Prevención, 99, I21128.

Sousa,V., Driessnack, M., \& Costa, I.A. (2007). Revisión de diseños de investigación resaltantes para enfermería. Parte I: diseños de investigación cuantitativa. Revista Latino-Americana de Enfermagem, 3(15).

Uriarte, J. D. (2006). Construir la resiliencia en la escuela. Revista de Psicodidáctica, I I(I), 7-24. 